\title{
Maternal intake of sugar during pregnancy and childhood respiratory and atopic outcomes
}

\author{
Annabelle Bédard ${ }^{1}$, Kate Northstone ${ }^{2,3}$, A. John Henderson ${ }^{3,4}$ and \\ Seif O. Shaheen ${ }^{1,4}$
}

Affiliations: ${ }^{1}$ Centre for Primary Care and Public Health, Barts and The London School of Medicine and Dentistry, Queen Mary University of London, London, UK. ${ }^{2}$ National Institute for Health Research Collaboration for Leadership in Applied Health Research and Care West, Bristol, UK. ${ }^{3}$ School of Social and Community Medicine, University of Bristol, Bristol, UK. ${ }^{4}$ Joint senior authors.

Correspondence: Annabelle Bédard, Centre for Primary Care and Public Health, Blizard Institute, Barts and The London School of Medicine and Dentistry, 58 Turner Street, London, E1 2AB, UK. E-mail: a.bedarddamul.ac.uk

@ERSpublications

Higher maternal intake of sugar in pregnancy may increase the risk of allergy and allergic asthma in the offspring http://ow.ly/zehc30bFswP

Cite this article as: Bédard $\mathrm{A}$, Northstone $\mathrm{K}$, Henderson $\mathrm{AJ}$, et al. Maternal intake of sugar during pregnancy and childhood respiratory and atopic outcomes. Eur Respir J 2017; 50: 1700073 [https://doi.org/ 10.1183/13993003.00073-2017].

ABSTRACT The possible role of maternal consumption of free sugar during pregnancy in the inception of respiratory and atopic diseases has not been studied. We aimed to study the relationship between maternal intake of free sugar during pregnancy and respiratory and atopic outcomes in the offspring in a population-based birth cohort, the Avon Longitudinal Study of Parents and Children.

We analysed associations between maternal intake of free sugar in pregnancy (estimated by a food frequency questionnaire), and current doctor-diagnosed asthma, wheezing, hay fever, eczema, atopy, serum total IgE and lung function in children aged $7-9$ years $(n=8956$ with information on maternal diet in pregnancy and at least one outcome of interest).

After controlling for potential confounders, maternal intake of free sugar was positively associated with atopy (OR for highest versus lowest quintile of sugar intake 1.38, 95\% CI 1.06-1.78; per quintile p-trend=0.006) and atopic asthma (OR 2.01, 95\% CI 1.23-3.29; per quintile p-trend=0.004). These associations were not confounded by intake of sugar in early childhood, which was unrelated to these outcomes.

Our results suggest that a higher maternal intake of free sugar during pregnancy is associated with an increased risk of atopy and atopic asthma in the offspring, independently of sugar intake in early childhood.

This article has supplementary material available from erj.ersjournals.com

Received: Jan 132017 | Accepted after revision: May 052017

Support statement: The UK Medical Research Council, the Wellcome Trust (Grant 102215/2/13/2) and the University of Bristol currently provide core support for the Avon Longitudinal Study of Parents and Children. A. Bédard is funded by a European Respiratory Society Long-Term Research Fellowship (LTRF 2015-5838). K. Northstone is supported by the National Institute for Health Research Collaboration for Leadership in Applied Health Research and Care West at University Hospitals Bristol NHS Foundation Trust. Funding information for this article has been deposited with the Crossref Funder Registry.

Conflict of interest: Disclosures can be found alongside this article at erj.ersjournals.com

Copyright @ERS 2017. This version is distributed under the terms of the Creative Commons Attribution Licence 4.0. 


\section{Introduction}

There has been considerable interest in the role of maternal diet in pregnancy in the aetiology of childhood asthma and atopy [1]. Studies have focused particularly on the potentially beneficial effects of antioxidants, following the hypothesis that a declining intake of antioxidants in Westernised countries has led to a reduction in pulmonary antioxidant defences, and hence to an increase in prevalence of asthma and atopy in recent decades [2]. An alternative hypothesis, which has received less attention, is that the epidemic of asthma and atopy in the West could partly be explained by an increasing dietary intake of foods and constituents which may be harmful. Between 1970 and 2000, there was a 25\% increase in the per capita consumption of all refined sugars in the USA, matching a worldwide trend [3]. Current international dietary guidelines advise people to reduce their consumption of sugar, and more particularly free sugars, which comprise sugars (monosaccharides and disaccharides) added to foods or drinks by the manufacturer, cook or consumer, and sugars naturally present in honey, syrups and unsweetened fruit juices [4]. While in children a high consumption of sugar-sweetened beverages [5-7] and fruit juice [7, 8] has been linked to asthma, and particularly atopic asthma [7], the relation between total maternal consumption of free sugar during pregnancy and respiratory and atopic outcomes in the offspring has not been studied. One ecological study reported a correlation between perinatal consumption of sugar and severe childhood asthma symptoms [9], but could not specifically address maternal sugar intake in pregnancy. A recent Danish birth cohort study investigated the relation between soft drink consumption, but not total free sugar intake, during pregnancy and childhood asthma and allergic rhinitis [10].

We have investigated whether a high intake of free sugar in pregnancy is associated with adverse respiratory and atopic outcomes in the offspring in a large population-based UK birth cohort.

\section{Methods \\ Participants}

The Avon Longitudinal Study of Parents and Children (ALSPAC) is a population-based birth cohort that recruited 14541 predominantly white pregnant women resident in Avon, UK with expected dates of delivery from April 1, 1991 to December 31, 1992. These pregnancies resulted in 13972 singleton or twin children who were alive at 1 year of age. The cohort has been followed since birth with annual questionnaires and, since age 7 years, with objective measures in annual research clinics. The study protocol has been described previously $[11,12]$ and further information can be found at www.alspac.bris. ac.uk, which contains details of all the data that are available (www.bris.ac.uk/alspac/researchers/ data-access/data-dictionary/). Ethics approval was obtained from the ALSPAC Ethics and Law Committee (IRB 00003312) and the Local National Health Service Research Ethics Committees.

\section{Exposure assessment}

Data on maternal diet in pregnancy were collected by a food frequency questionnaire (FFQ) at 32 weeks gestation, covering all the main foods consumed in Britain [13]. The questionnaire asked about their current weekly frequency of consumption of 43 food groups and food items. More detailed questions were asked about daily consumption of a further eight basic foods (including sugar, coffee and tea). The FFQ was used to estimate total energy intake and daily nutrient intake, by multiplying the daily frequency of consumption of a food by the nutrient content [14] of a standard portion [15] of that food, and summing this for all the foods consumed. In this way free sugar intake was estimated. Free sugar does not include lactose when naturally present in milk and milk products or the sugars contained within the cellular structure of fruits and vegetables. Information on the child's free sugar consumption at age 3 years, as well as maternal and paternal sugar consumption at 4 years post-partum, was collected by a similar FFQ.

Information from a questionnaire at recruitment and from the obstetric records was used to classify women into four mutually exclusive categories: no evidence of glycosuria or diabetes, existing diabetes mellitus before the pregnancy, gestational diabetes and persistent glycosuria during pregnancy. For the purposes of analysis we combined the last three categories to create a binary maternal "diabetes" variable (see supplementary material for further details).

\section{Outcome assessment}

Binary variables

Current doctor-diagnosed asthma was defined in children at age 7.5 years (primary outcome) if mothers responded positively to the question "Has a doctor ever actually said that your study child has asthma?" and to one or both of the questions "Has your child had any of the following in the past 12 months: wheezing with whistling; asthma?" 
Current wheezing, eczema and hay fever in children at age 7.5 years were defined by a positive answer to the question: "Has your child had any of the following in the past 12 months: wheezing with whistling; eczema; hay fever?"

Atopy at age 7 years was defined as a positive reaction (maximum diameter of any detectable weal) to Dermatophagoides pteronyssinus, cat or grass (after subtracting positive saline reactions from histamine and allergen weals, and excluding children unreactive to $1 \%$ histamine).

\section{Categorical variables}

Children were further classified, post hoc, according to their asthmatic/atopic status, thus defining a four-category variable (no atopy or asthma, atopy only, nonatopic asthma and atopic asthma), and according to their number of positive reactions to cat, grass and dust mite allergens $(n=0,1$ and $\geqslant 2$ ).

Data on child's asthma status at age 7 and 14 years were used to derive asthma status phenotypes between age 7 and 14 years (none, remitting, incident and persisting) [16].

\section{Continuous variables}

Serum total IgE $\left(\mathrm{kU} \cdot \mathrm{L}^{-1}\right)$ was measured by fluoroimmunoassay using the Pharmacia UNICAP system (Pharmacia and Upjohn Diagnostics, Uppsala, Sweden).

Lung function was measured by spirometry (Vitalograph 2120; Vitalograph, Maids Moreton, UK) at age 8.5 years after withholding short-acting bronchodilators for at least $6 \mathrm{~h}$ and long-acting bronchodilators and theophyllines for at least $24 \mathrm{~h}$. The best of three reproducible flow-volume curves was used to measure forced expiratory volume in $1 \mathrm{~s}(\mathrm{FEV} 1)$, forced vital capacity (FVC) and maximal mid-expiratory flow (forced expiratory flow at $25-75 \%$ of FVC (FEF25-75)), which were further transformed to age-, height- and sex-adjusted standard deviation units [17]. The tests adhered to American Thoracic Society (ATS) criteria for standardisation and reproducibility of flow-volume measurement [18], with the exception of ATS recommendations for duration of expiration [19]; as many children did not fulfil forced expiratory time $>6 \mathrm{~s}$ end-of-test criteria, a minimal volume change over the final $1 \mathrm{~s}$ was used.

\section{Potential confounders}

We selected potential confounding factors which are known (from existing literature) to be associated with one or more of the outcomes of interest [20]. These included maternal age at delivery, sex of child, multiple pregnancy, season of birth, maternal history of atopic diseases (hay fever, asthma, eczema, allergies, or attacks of wheezing with whistling on the chest or attacks of breathlessness in the past 2 years), parity, highest educational qualification, housing tenure, financial difficulties, ethnicity, breastfeeding duration and maternal factors during pregnancy (smoking status, anxiety score (CrownCrisp Experiential Index), paracetamol use, antibiotic use, infections (urinary infection, influenza, rubella, thrush, genital herpes, other), supplement use and total energy intake $\left(\mathrm{kJ} \cdot \mathrm{day}^{-1}\right)$ ). Smoking status was categorised as the maximum exposure during pregnancy (never, passive smoking only, 1-9, 10-19 and $\geqslant 20$ cigarettes per day).

\section{Statistical analyses}

We compared the distributions of child and maternal variables across maternal free sugar intake quintiles using F-statistics for differences in continuous variables and Chi-squared tests for differences in categorical variables. Logistic regression, multinomial logistic regression and linear regression were used to analyse relations between maternal free sugar intake in pregnancy and binary, categorical and continuous outcomes, respectively. After log-transforming total IgE, linear regression was used to estimate geometric mean ratios for IgE; confidence limits were calculated using Huber variances. We analysed free sugar intake in quintiles: 1) as a categorical variable using the lowest quintile as reference to allow for a nonlinear pattern of association and 2) as a continuous variable to test for linear trend (i. $e$. per quintile effect). For all regression analyses, two stages of adjustment were used. In Model 1 we adjusted for total energy intake only. In Model 2 we adjusted additionally for all potential confounders listed above.

When evidence for associations persisted, we considered other factors which can be considered either as potential confounders or potential mediators of associations between maternal free sugar intake in pregnancy and childhood outcomes, i.e. prematurity [21, 22], impaired fetal growth [21, 23], maternal obesity and weight gain [24-26], and offspring obesity [27, 28]. We therefore adjusted additionally for maternal pre-pregnancy body mass index (BMI) (self-reported), gestational age at delivery, birthweight, maternal weight gain during pregnancy (all abstracted from obstetric records) and child's BMI at age 7 years (based on measured height and weight at clinic) (see supplementary figure E1 showing a directed acyclic graph). In order to assess confounding arising from other 
TABLE 1 Characteristics of mothers and offspring who had information on at least one of the outcomes of interest (wheeze, asthma, atopy, eczema, hay fever, total $\lg E$ and lung function) by maternal free sugar intake during pregnancy ${ }^{\#}$

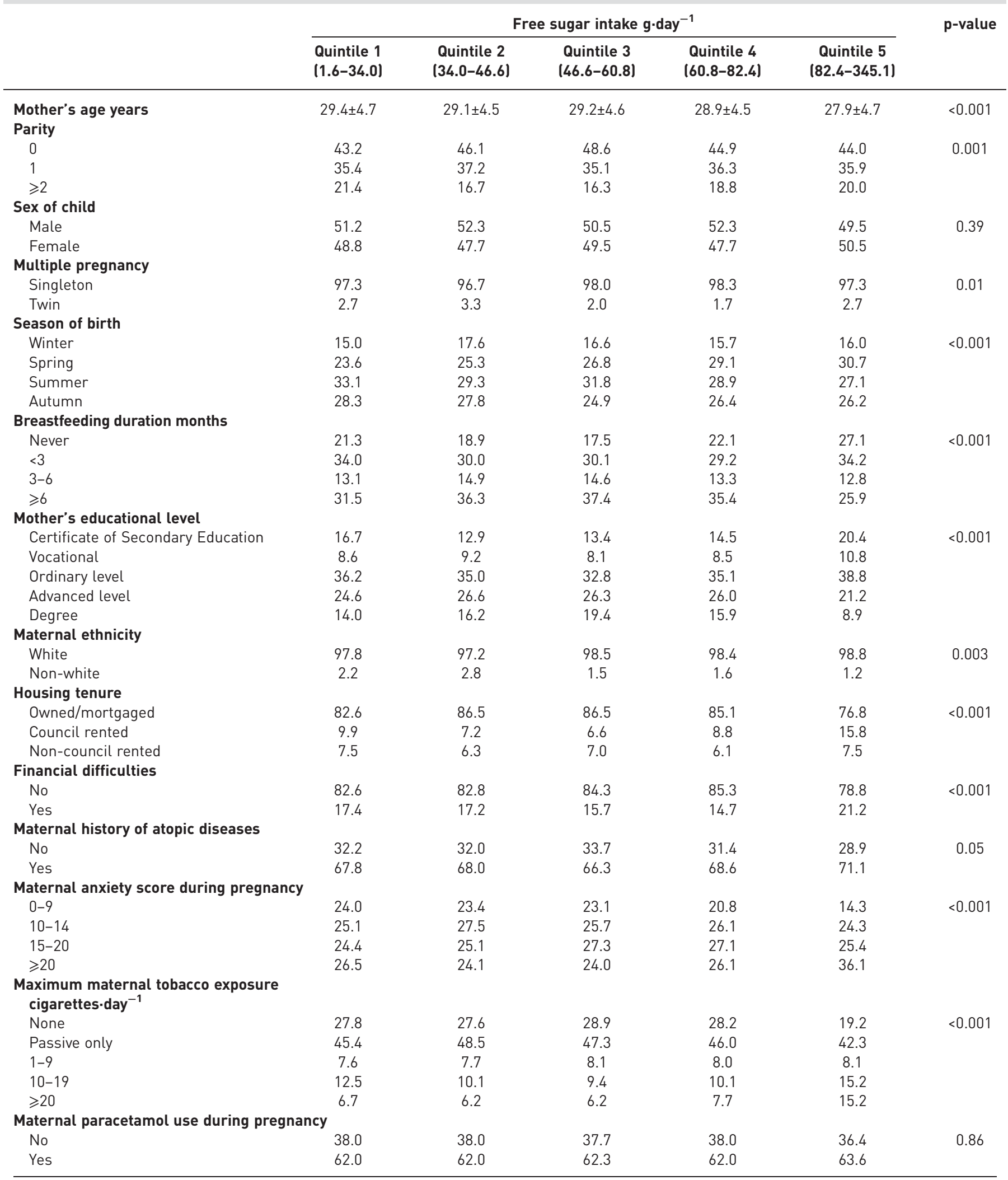


TABLE 1 Continued

\begin{tabular}{|c|c|c|c|c|c|c|}
\hline & \multicolumn{5}{|c|}{ Free sugar intake $\mathbf{g} \cdot$ day $^{-1}$} & \multirow[t]{2}{*}{ p-value } \\
\hline & $\begin{array}{l}\text { Quintile } 1 \\
(1.6-34.0)\end{array}$ & $\begin{array}{l}\text { Quintile } 2 \\
(34.0-46.6)\end{array}$ & $\begin{array}{l}\text { Quintile } 3 \\
(46.6-60.8)\end{array}$ & $\begin{array}{l}\text { Quintile } 4 \\
(60.8-82.4)\end{array}$ & $\begin{array}{c}\text { Quintile } 5 \\
(82.4-345.1)\end{array}$ & \\
\hline \multicolumn{7}{|c|}{ Maternal antibiotic use during pregnancy } \\
\hline No & 83.7 & 85.4 & 84.0 & 83.7 & 82.9 & 0.34 \\
\hline Yes & 16.3 & 14.6 & 16.0 & 16.3 & 17.1 & \\
\hline \multicolumn{7}{|c|}{$\begin{array}{l}\text { Maternal supplement use during } \\
\text { pregnancy }\end{array}$} \\
\hline Yes & 55.5 & 56.9 & 55.6 & 58.1 & 58.4 & \\
\hline \multicolumn{7}{|c|}{ Maternal infections during pregnancy } \\
\hline No & 57.2 & 55.1 & 55.8 & 53.3 & 49.3 & $<0.001$ \\
\hline Yes & 42.8 & 44.9 & 44.2 & 46.7 & 50.7 & \\
\hline Total energy intake $\mathbf{k J} \cdot$ day $^{-1}$ & $5567 \pm 1376$ & $6499 \pm 1326$ & $7166 \pm 1360$ & $7931 \pm 1488$ & $9317 \pm 1994$ & $<0.001$ \\
\hline \multicolumn{7}{|c|}{ Maternal pre-pregnancy BMI $\mathrm{kg} \cdot \mathrm{m}^{-2}$} \\
\hline$<2500$ & 4.2 & 4.0 & 3.6 & 4.3 & 5.6 & 0.02 \\
\hline $2500-2999$ & 14.2 & 13.2 & 13.9 & 12.8 & 15.2 & \\
\hline $3000-3499$ & 34.8 & 34.9 & 35.1 & 36.7 & 35.6 & \\
\hline $3500-3999$ & 32.2 & 33.6 & 33.5 & 33.6 & 33.0 & \\
\hline$\geqslant 4000$ & 14.7 & 14.4 & 13.8 & 12.6 & 11.0 & \\
\hline Gestational age weeks & $39.4 \pm 1.8$ & $39.5 \pm 1.7$ & $39.5 \pm 1.7$ & $39.5 \pm 1.8$ & $39.4 \pm 1.9$ & 0.28 \\
\hline \multicolumn{7}{|c|}{ Child's BMI at age 7 years $\mathrm{kg} \cdot \mathrm{m}^{-2}$} \\
\hline$<15.00$ & 25.8 & 26.3 & 30.3 & 27.7 & 30.7 & $<0.001$ \\
\hline $15.00-17.49$ & 51.9 & 52.3 & 51.2 & 54.4 & 52.6 & \\
\hline $17.50-20.49$ & 17.2 & 16.6 & 14.2 & 14.3 & 13.6 & \\
\hline$\geqslant 20.50$ & 5.2 & 4.8 & 4.3 & 3.5 & 3.1 & \\
\hline \multicolumn{7}{|c|}{ Maternal weight gain during pregnancy } \\
\hline
\end{tabular}

p-value

Data are presented as mean \pm SD or $\%$, unless otherwise stated. BMI: body mass index. ${ }^{\#}: \mathrm{n}=8956$.

dimensions of diet, we additionally adjusted separately for maternal intake of vitamin E, zinc, selenium, n-3 polyunsaturated fatty acids (PUFAs), n-6 PUFAs, and total fruits and vegetables in pregnancy [1, 29-31].

To investigate confounding by post-natal sugar intake, we adjusted additionally for child's sugar intake at age 3 years. In order to investigate potential unmeasured confounding by genetic or shared environmental or lifestyle factors, we used a parental comparison approach, whereby effect estimates for maternal sugar intake in pregnancy were compared with effect estimates for maternal and paternal sugar intake after pregnancy. If there is a causal intrauterine effect, one would expect a stronger association with maternal intake in pregnancy than with maternal post-natal intake or paternal intake (the latter two exposures cannot have a direct biological effect on offspring asthma risk) (see further details in the supplementary material) $[32,33]$.

As sensitivity analyses, we repeated analyses after exclusion of mothers with implausible energy intakes $\left(<2500\right.$ or $>25000 \mathrm{~kJ} \cdot \mathrm{day}^{-1}$ [34]) and after exclusion of mothers with diabetes (whose offspring will have experienced high fetal exposure to glucose). To correct for potential loss to follow-up bias, we used inverse probability weighting and assigned to each woman a weight that was the inverse of the probability of her selection for given values of covariates (see further details in the supplementary material) [35]. All statistical analyses were carried out using Stata version 12.1 (StataCorp, College Station, TX, USA). 


\section{Results}

Of the 13972 singleton or twin children alive at 1 year of age, information on maternal diet was available for 12078, of whom there was information on at least one of the outcomes of interest for 8956 (supplementary figure E2). Characteristics of the 8956 mother-child pairs who were included in the analyses and those of the 3122 mother-child pairs with information on maternal diet who were excluded because of incomplete outcome data are compared in supplementary table E1. Among children with available information, $12.2 \%$ had current doctor-diagnosed asthma, $10.7 \%$ had current wheezing with whistling, $8.8 \%$ had current hay fever, $16.2 \%$ had current eczema, $21.5 \%$ had atopy and $61.8 \%$ did not have any of these five outcomes.

Maternal characteristics which differed across quintiles of free sugar intake during pregnancy included age, parity, pregnancy size, season of birth, breastfeeding duration, educational level, ethnicity, housing tenure, financial difficulties, anxiety level, tobacco exposure and infection during pregnancy. Women in the highest quintile of total sugar intake during pregnancy had a lower pre-pregnancy BMI, higher total energy intake and gained more weight during pregnancy than women in the lowest quintile. Their offspring were more likely to have weighed less at birth and to have had a lower BMI at age 7 years (table 1). After adjustment for potential confounders, there was weak evidence for positive associations between maternal free sugar intake in pregnancy and childhood doctor-diagnosed asthma and childhood wheeze (OR comparing highest versus lowest quintile $1.31,95 \%$ CI $0.98-1.75$; per quintile p-trend $=0.09$ and $1.42,95 \%$ CI 1.05-1.92; per quintile p-trend $=0.08$, respectively), and stronger evidence for a positive association with atopy at age 7 years (OR 1.38, 95\% CI 1.06-1.78; per quintile p-trend=0.006) (table 2). There was no association with eczema, hay fever, total IgE, FEV1, FVC or FEF25-75 (table 2 and supplementary table E2). Post hoc analysis showed a positive association between maternal intake of free sugar and atopic asthma (OR 2.01, 95\% CI 1.23-3.29; per quintile p-trend=0.004) (table 3). The main positive findings of our study are summarised in figure 1 .

Further investigation of potential confounding/mediation of main findings and sensitivity analyses Additional separate adjustment for maternal pre-pregnancy BMI, gestational age at delivery, birthweight, maternal weight gain during pregnancy and child's BMI at age 7 years did not substantially alter the main findings and therefore no further formal mediation analysis was conducted (supplementary table E3). Additional separate adjustment for maternal intake of vitamin E, zinc, selenium, n-3 PUFAs, n-6 PUFAs, and total fruits and vegetables in pregnancy did not substantially alter the main findings (data not shown), nor did additional separate adjustment for child's free sugar intake at age 3 years (supplementary table E3). The latter exposure was not associated with any outcome (data not shown).

TABLE 2 Associations between maternal free sugar intake during pregnancy and asthma, wheeze, eczema, hay fever and atopy in the offspring

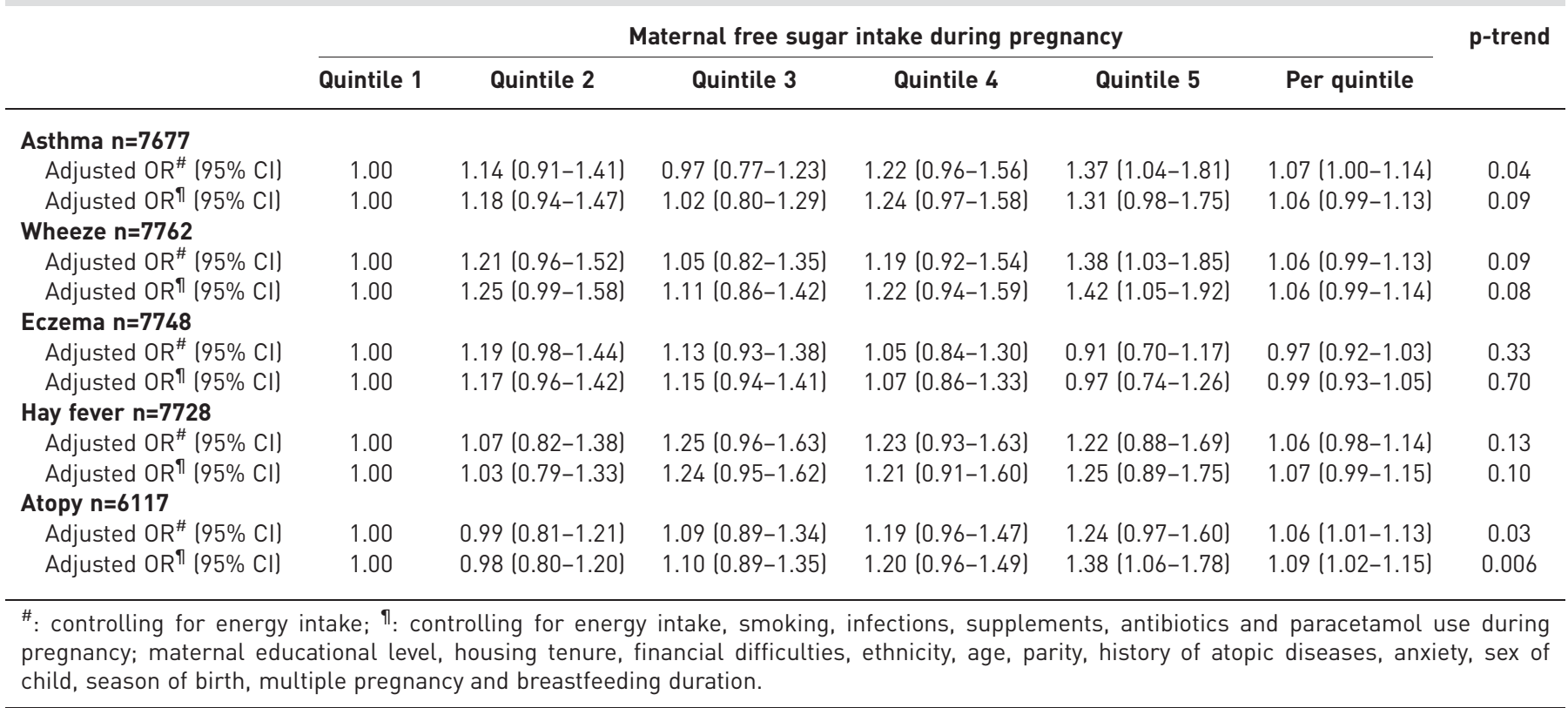


TABLE 3 Associations between maternal free sugar intake during pregnancy and atopy without asthma, nonatopic and atopic asthma ${ }^{\#}$ in the offspring

\begin{tabular}{|c|c|c|c|c|c|c|c|}
\hline & \multicolumn{6}{|c|}{ Maternal free sugar intake during pregnancy } & p-trend \\
\hline \multicolumn{8}{|c|}{ Atopy without asthma $n=794$} \\
\hline Adjusted $\mathrm{OR}^{+}(95 \% \mathrm{Cl})$ & 1.00 & $0.77(0.60-0.99)$ & $1.05(0.82-1.34)$ & $0.95(0.72-1.25)$ & $1.17(0.85-1.62)$ & $1.05(0.98-1.13)$ & 0.19 \\
\hline \multicolumn{8}{|l|}{ Nonatopic asthma $\mathrm{n}=301$} \\
\hline Adjusted OR (95\% CI) & 1.00 & $0.82(0.57-1.16)$ & $0.67(0.46-0.99)$ & $0.77(0.52-1.16)$ & $0.83(0.52-1.34)$ & $0.95(0.85-1.06)$ & 0.34 \\
\hline Adjusted OR $(95 \%$ Cl) & 1.00 & $1.66(1.14-2.41)$ & $1.17(0.78-1.77)$ & $2.09(1.39-3.14)$ & $1.79(1.11-2.90)$ & $1.14(1.03-1.27)$ & 0.01 \\
\hline Adjusted $\mathrm{OR}^{+}(95 \% \mathrm{Cl})$ & 1.00 & $1.75(1.20-2.56)$ & $1.27(0.84-1.93)$ & $2.18(1.45-3.30)$ & $2.01(1.23-3.29)$ & $1.17(1.05-1.30)$ & 0.004 \\
\hline
\end{tabular}

In subsets of the cohort with complete data for paternal (respectively, maternal) free sugar intake after pregnancy, no association was found between paternal (respectively, maternal) free sugar intake after pregnancy and childhood atopy or atopic asthma. The significant associations of maternal free sugar intake during pregnancy with childhood atopy and atopic asthma remained, unattenuated, on mutual adjustment for paternal (respectively, maternal) post-natal exposure (table 4 and supplementary table E4, respectively).

When we analysed the association between maternal free sugar intake and the number of positive reactions to cat, grass and dust mite allergens, we observed a stronger association for children with two or more positive reactions (table 5). We studied associations between maternal free sugar intake in pregnancy and childhood asthma status phenotypes, and did not observe any association (supplementary table E5).

The exclusion of 17 women with implausible energy intake estimates did not alter the main results nor did exclusion of mothers with diabetes. Maternal "diabetes" was not associated with any respiratory or atopic outcome, but was associated with higher birthweight. The inverse probability weighting analysis also produced similar results (data not shown).
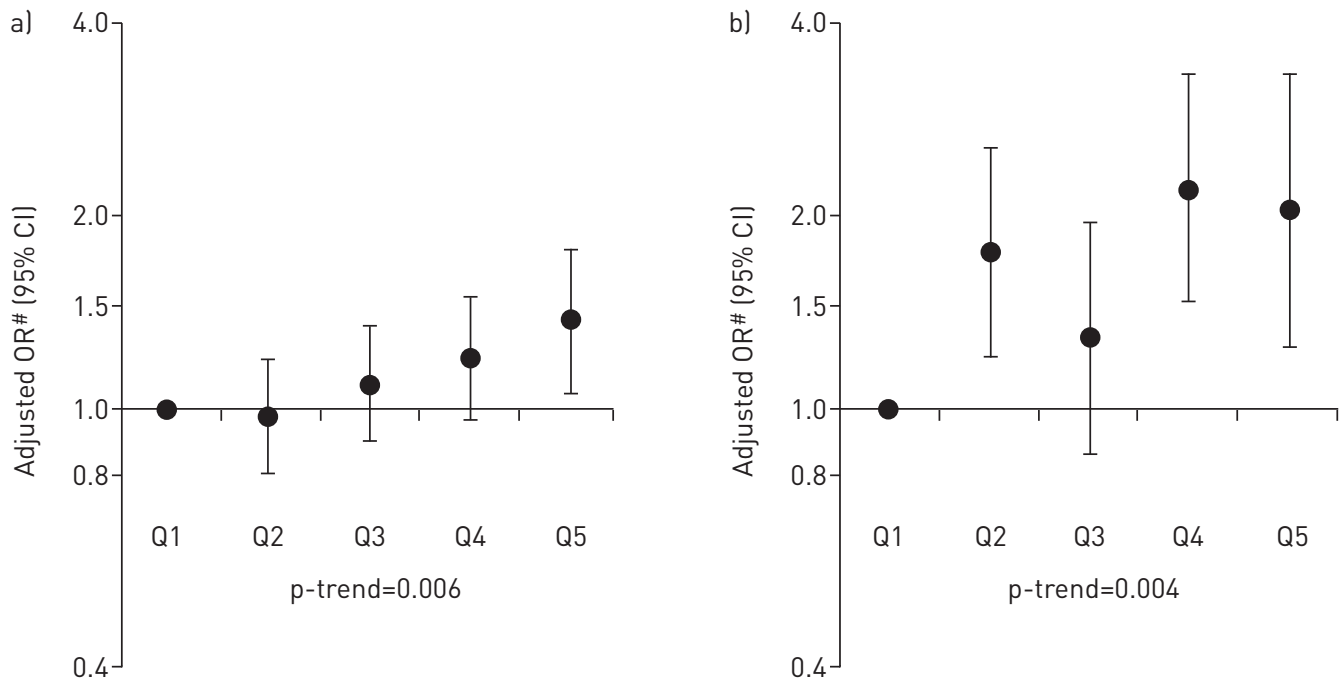

FIGURE 1 Summary of the main findings for the associations between maternal free sugar intake during pregnancy and childhood outcomes: a) atopy ( $n=6117)$ and b) atopic asthma ( $n=5228)$. Q: quintile. \#: controlling for energy intake, smoking, infections, supplements, antibiotics and paracetamol use during pregnancy; maternal educational level, housing tenure, financial difficulties, ethnicity, age, parity, history of atopic diseases, anxiety, sex of child, season of birth, multiple pregnancy and breastfeeding duration. 
TABLE 4 Comparison of associations of childhood atopy and atopic asthma with maternal free sugar intake during pregnancy versus paternal intake after pregnancy

Free sugar intake

p-trend

\begin{tabular}{llllll}
\hline Quintile 1 & Quintile 2 & Quintile 3 & Quintile 4 & Quintile 5 & Per quintile
\end{tabular}

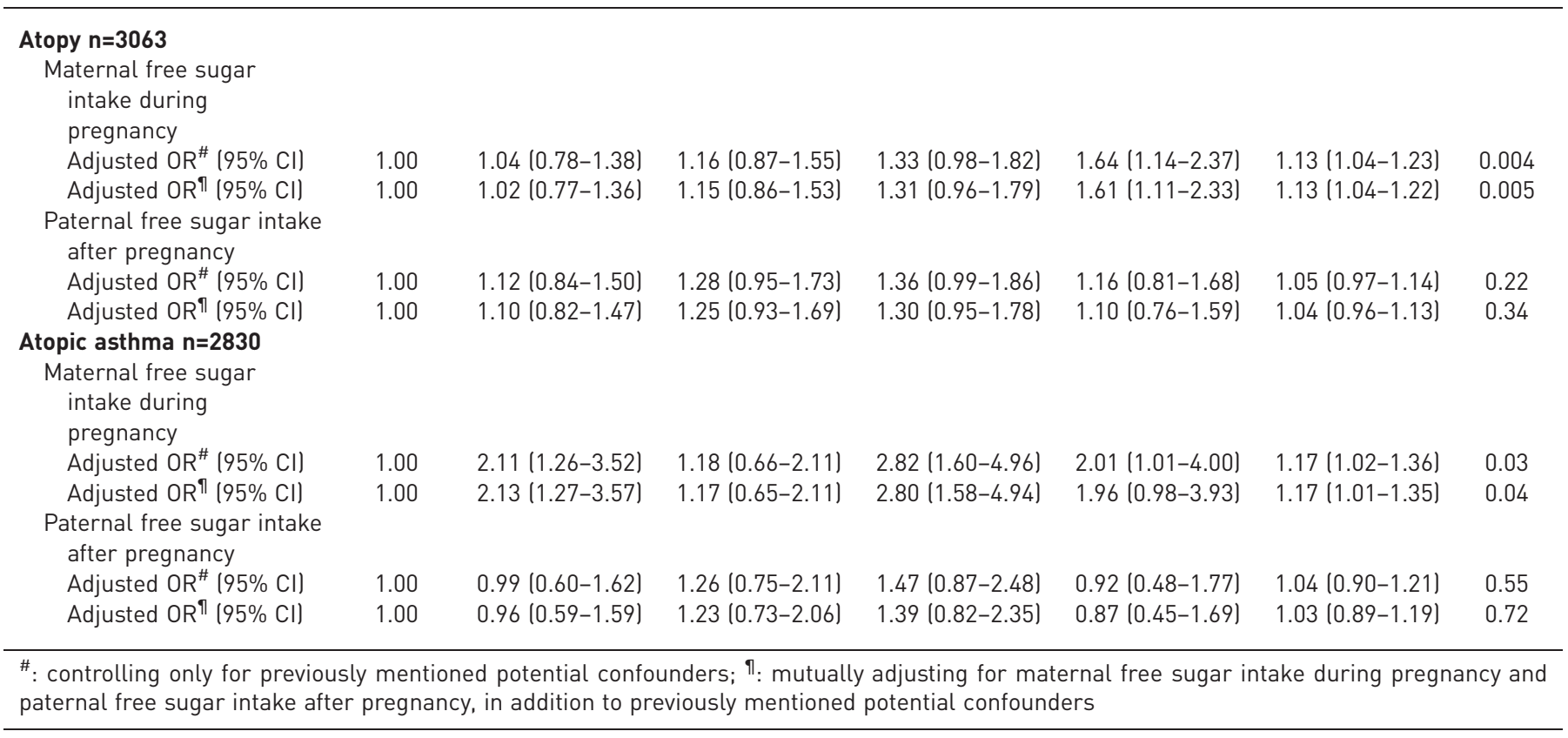

\section{Discussion}

In this population-based birth cohort study, we found that a higher maternal intake of free sugar during pregnancy was associated with an increased risk of atopy and atopic asthma in the offspring, independently of sugar intake in early childhood.

To the best of our knowledge, these are novel findings. While a previous ecological study reported a positive correlation between perinatal consumption of sugar and severe childhood asthma symptoms [9], the limitations of ecological studies for inferring causality are well known (not least because of the high likelihood of confounding) [36]. Furthermore, that study was unable to specifically investigate the potential role of maternal intake of sugar during pregnancy nor the specific role of free sugar. Our longitudinal findings linking maternal free sugar intake in pregnancy to childhood atopy and atopic asthma extend the ecological results and allow stronger causal inference. Interestingly, the findings for atopy became stronger when we examined the association with multiple sensitisation. Although previous cross-sectional studies

TABLE 5 Associations between maternal free sugar intake in pregnancy and number of positive skin prick tests (SPTs)

\begin{tabular}{|c|c|c|c|c|}
\hline \multirow[t]{2}{*}{ Free sugar } & \multicolumn{2}{|c|}{1 positive SPT ${ }^{\Uparrow}$} & \multicolumn{2}{|c|}{$\geqslant 2$ positive SPTs ${ }^{+}$} \\
\hline & Adjusted $\mathrm{OR}^{\S}(95 \% \mathrm{CI})$ & p-trend & Adjusted $\mathrm{OR}^{\S}(95 \% \mathrm{CI})$ & p-trend \\
\hline Quintile 1 & 1.00 & & 1.00 & \\
\hline Quintile 2 & $0.87(0.68-1.12)$ & & 1.17 (0.87-1.57) & \\
\hline Quintile 3 & $1.07(0.83-1.37)$ & & $1.14(0.84-1.56)$ & \\
\hline Quintile 4 & $1.09(0.83-1.43)$ & & $1.37(0.99-1.90)$ & \\
\hline Quintile 5 & $1.19(0.86-1.63)$ & & $1.73(1.18-2.52)$ & \\
\hline Per quintile & $1.06(0.98-1.14)$ & 0.12 & $1.13(1.03-1.23)$ & 0.006 \\
\hline
\end{tabular}

\#: no positive SPT was considered as baseline category $(n=4797) ;{ }^{\uparrow}: n=783 ;{ }^{+}: n=531 ;{ }^{\S}$ : controlling for energy intake, smoking, infections, supplements, antibiotics and paracetamol use during pregnancy; maternal educational level, housing tenure, financial difficulties, ethnicity, age, parity, history of atopic diseases, anxiety, sex of child, season of birth, multiple pregnancy and breastfeeding duration. 
have reported a positive association between childhood consumption of sugar-containing drinks, including fruit juice, and asthma [5-8], intake of free sugar in early childhood in our study was not associated with any respiratory or atopic outcome.

\section{Mechanisms}

We speculate that high maternal fructose consumption may underlie the positive associations between maternal intake of free sugar and childhood atopy and atopic asthma. Fructose, which is a major component of added sugars, and is present naturally in fruit juice and in sweetened drinks as added sucrose (ratio of fructose/glucose 50/50\%) or isolated fructose, has been mooted as driving previous cross-sectional findings linking sugar-containing beverage consumption to asthma in children $[7,8]$. Fructose consumption, in the form of high fructose corn syrup (ratio of fructose/glucose 60/40\%), increased from near $0 \%$ to near $30 \%$ of per capita consumption of refined sugars in the USA between 1970 and 2000, whereas the consumption of sucrose and glucose declined or remained constant [3].

A prospective randomised controlled trial in adults showed that dietary sugar, and especially fructose, increased levels of C-reactive protein [37]. Fructose also causes generation of uric acid [38], and experimental evidence in mice suggests that uric acid may be an essential initiator and amplifier of T-helper cell type 2 (Th2) immunity and allergic inflammation, through activation of inflammatory dendritic cells [39]. Alternatively, fructose might influence atopic immune responses by conditioning the gut microbiome [40,41]. The potential of maternal diet in pregnancy to influence inception of offspring allergic airways disease through this mechanism was recently confirmed in a mouse model [42]. We therefore propose that one explanation for our main findings is that high fetal exposure to fructose may cause persistence of Th2 immune responses post-natally and allergic inflammation in the developing lung.

In contrast to a previous study which reported a link between gestational diabetes and risk of atopic eczema and atopy in early childhood [43], we found no association between maternal diabetes during pregnancy and any outcome in the offspring, although, as expected [44], maternal diabetes was associated with higher birthweight. The lack of a relation with maternal diabetes would suggest that higher fetal exposure to glucose is unlikely to explain our main findings. While high fructose consumption has been proposed as a risk factor for obesity [45], we found no evidence to suggest that the associations between maternal free sugar intake and atopy and atopic asthma in the offspring were mediated by maternal BMI, gestational weight gain or child's BMI, nor by prematurity or low birthweight, assuming key assumptions necessary for mediation analyses were met $[46,47]$.

\section{Strengths and limitations}

Strengths of the ALSPAC birth cohort include its size and population-based prospective design, rich information on numerous potential lifestyle and dietary confounders (including information on childhood free sugar intake and parental sugar intake outside of pregnancy), and detailed phenotypic outcome measurements.

Although the FFQ that we used had not been formally calibrated against other instruments such as diet diaries, it was based on the one used by YARNELL et al. [48], which has been validated against weighed dietary records and modified in the light of a more recent weighed dietary survey [13]. The FFQ lacked quantitative information on soft drink consumption and this will have led to underestimation of maternal free sugar intake during pregnancy. However, as misclassification of maternal free sugar intake in pregnancy is likely to have been random with respect to childhood outcomes, the strength of associations may have been underestimated. We were unable to assess associations with maternal sugar intake in early pregnancy; however, intakes in early and late pregnancy are likely to be highly correlated. We were unable to assess whether associations between maternal intake of free sugar in pregnancy and childhood atopy and atopic asthma persist beyond the age of 7 years, as no data on atopy (only data on asthma status) have been collected in ALSPAC children after the age of 7 years.

We think that confounding of the main findings by lifestyle or other aspects of maternal diet in pregnancy is unlikely, as we controlled for numerous potential confounders in the analyses, including nutrients and foods that have been previously linked to childhood asthma and atopy. Importantly, the main findings were not confounded by the offspring's free sugar intake in early childhood. While the possibility of residual confounding cannot be ruled out, the null findings for maternal and paternal free sugar intakes after pregnancy make confounding by unmeasured familial behaviours linked to sugar intake and asthma risk a less likely explanation.

As with any longitudinal study, we cannot rule out the possibility that exclusion of mother-child pairs without complete information might have biased our findings. However, it could be argued that, for our results to be totally spurious in those included in our analysis (and for the associations to be truly null in 
the population as a whole), associations in the excluded mother-child pairs would have to be in the opposite direction and much stronger, compared with the positive associations we reported in the included mother-child pairs, which seems extremely unlikely. Furthermore, loss to follow-up bias has been shown to only slightly modify associations in longitudinal studies, including in ALSPAC [49], and the results of our inverse probability weighting analysis [35] confirmed that loss to follow-up is unlikely to have biased our results. In view of the multiple analyses carried out and the post hoc nature of the findings for atopic asthma, we cannot exclude the possibility that the main findings occurred by chance; hence, they should be interpreted with caution. Given the a priori nature of the hypothesis being tested, and the fact that some outcomes of interest are highly correlated, it did not seem appropriate to correct for multiple testing. However, we plan to re-examine this hypothesis in another birth cohort to see if we can replicate the main findings.

\section{Conclusions and public health implications}

We conclude that a higher maternal intake of free sugar during pregnancy may increase the risk of atopy and atopic asthma in the offspring. If these findings are replicated we would design an appropriate intervention study in pregnancy to establish or refute causality. Given the very high levels of sugar consumption currently in the West, where childhood allergy and asthma are so prevalent, confirmation of a causal link would raise exciting prospects for the primary prevention of these disorders.

\section{Acknowledgements}

We are extremely grateful to all the families who took part in this study, the midwives for their help in recruiting them, and the whole Avon Longitudinal Study of Parents and Children team, which includes interviewers, computer and laboratory technicians, clerical workers, research scientists, volunteers, managers, receptionists, and nurses. The authors would like to thank especially Raquel Granell (School of Social and Community Medicine, University of Bristol, Bristol, UK) for preliminary analysis and data collection. This paper is the work of the authors, and A.J. Henderson and S.O. Shaheen will serve as guarantors for its contents. The views expressed are those of the author(s) and not necessarily those of the National Health Service, the National Institute for Health Research or the Dept of Health.

Author contributions: A. Bédard and S.O. Shaheen conceived the study and drafted the manuscript. All authors were involved in the analysis strategy, K. Northstone gave advice on the dietary data and A. Bédard performed the statistical analyses. A.J. Henderson was responsible for all clinical respiratory and allergy data collection. All authors participated in the interpretation of the findings, reviewed the manuscript and revised it critically before submission. All authors have seen and approved the final version of the manuscript.

\section{References}

1 Nurmatov U, Devereux G, Sheikh A. Nutrients and foods for the primary prevention of asthma and allergy: systematic review and meta-analysis. J Allergy Clin Immunol 2011; 127: 724-733.

2 Seaton A, Godden DJ, Brown K. Increase in asthma: a more toxic environment or a more susceptible population? Thorax 1994; 49: 171-174.

3 Cordain L, Eaton SB, Sebastian A, et al. Origins and evolution of the Western diet: health implications for the 21st century. Am J Clin Nutr 2005; 81: 341-354.

4 Scientific Advisory Committee on Nutrition. SCAN Carbohydrates and Health Report: the Scientific Advisory Committee on Nutrition's recommendations on carbohydrates, including sugars and fibre. 2015. www.gov.uk/ government/publications/sacn-carbohydrates-and-health-report Date last accessed: May 30, 2017.

5 Stensson M, Wendt L-K, Koch G, et al. Oral health in pre-school children with asthma - followed from 3 to 6 years. Int J Paediatr Dent 2010; 20: 165-172.

6 Park S, Blanck HM, Sherry B, et al. Regular-soda intake independent of weight status is associated with asthma among US high school students. J Acad Nutr Diet 2013; 113: 106-111.

7 Berentzen NE, van Stokkom VL, Gehring U, et al. Associations of sugar-containing beverages with asthma prevalence in 11-year-old children: the PIAMA birth cohort. Eur J Clin Nutr 2015; 69: 303-308.

8 DeChristopher LR, Uribarri J, Tucker KL. Intakes of apple juice, fruit drinks and soda are associated with prevalent asthma in US children aged 2-9 years. Public Health Nutr 2015; 19: 1-8.

9 Thornley S, Stewart A, Marshall R, et al. Per capita sugar consumption is associated with severe childhood asthma: an ecological study of 53 countries. Prim Care Respir J 2011; 20: 75-78.

10 Maslova E, Strøm M, Olsen SF, et al. Consumption of artificially-sweetened soft drinks in pregnancy and risk of child asthma and allergic rhinitis. PLoS One 2013; 8: e57261.

11 Boyd A, Golding J, Macleod J, et al. Cohort profile: the "Children of the 90s" - the index offspring of the Avon Longitudinal Study of Parents and Children. Int J Epidemiol 2013; 42: 111-127.

12 Fraser A, Macdonald-Wallis C, Tilling K, et al. Cohort profile: the Avon Longitudinal Study of Parents and Children: ALSPAC mothers cohort. Int J Epidemiol 2013; 42: 97-110.

13 Rogers I, Emmett P. Diet during pregnancy in a population of pregnant women in South West England. ALSPAC Study Team. Avon Longitudinal Study of Pregnancy and Childhood. Eur J Clin Nutr 1998; 52: 246-250.

14 McCance RA, Widdowson EM. The Composition of Foods. 5th Edn. London, Royal Society of Chemistry/ Ministry of Agriculture, Fisheries and Food, 1991.

15 Ministry of Agriculture, Fisheries and Food. Food Portion Sizes. London, HMSO, 1991.

16 Granell R, Henderson AJ, Sterne JA. Associations of wheezing phenotypes with late asthma outcomes in the Avon Longitudinal Study of Parents and Children: a population-based birth cohort. J Allergy Clin Immunol 2016; 138: $1060-1070$. 
17 Chinn S, Rona RJ. Height and age adjustment for cross sectional studies of lung function in children aged 6-11 years. Thorax 1992; 47: 707-714.

18 American Thoracic Society. Standardization of Spirometry, 1994 Update. Am J Respir Crit Care Med 1995; 152: 1107-1136.

19 Arets HGM, Brackel HJL, van der Ent CK. Forced expiratory manoeuvres in children: do they meet ATS and ERS criteria for spirometry? Eur Respir J 2001; 18: 655-660.

20 Nurmatov U, Nwaru BI, Devereux G, et al. Confounding and effect modification in studies of diet and childhood asthma and allergies. Allergy 2012; 67: 1041-1059.

21 Lenders CM, Hediger ML, Scholl TO, et al. Gestational age and infant size at birth are associated with dietary sugar intake among pregnant adolescents. J Nutr 1997; 127: 1113-1117.

22 Jaakkola JJK, Ahmed P, Ieromnimon A, et al. Preterm delivery and asthma: a systematic review and meta-analysis. J Allergy Clin Immunol 2006; 118: 823-830.

23 Brooks AM, Byrd RS, Weitzman M, et al. Impact of low birth weight on early childhood asthma in the United States. Arch Pediatr Adolesc Med 2001; 155: 401-406.

24 Tielemans MJ, Erler NS, Leermakers ETM, et al. A priori and a posteriori dietary patterns during pregnancy and gestational weight gain: the Generation R Study. Nutrients 2015; 7: 9383-9399.

25 Forno E, Young OM, Kumar R, et al. Maternal obesity in pregnancy, gestational weight gain, and risk of childhood asthma. Pediatrics 2014; 134: e535-e546.

26 Bédard A, Dumas O, Kauffmann F, et al. Potential confounders in the asthma-diet association: how causal approach could help? Allergy 2012; 67: 1461-1462.

27 Phelan S, Hart C, Phipps M, et al. Maternal behaviors during pregnancy impact offspring obesity risk. Exp Diabetes Res 2011; 2011: 985139.

28 Litonjua AA, Gold DR. Asthma and obesity: common early-life influences in the inception of disease. $J$ Allergy Clin Immunol 2008; 121: 1075-1084.

29 Varraso R. Nutrition and asthma. Curr Allergy Asthma Rep 2012; 12: 201-210.

30 Allan K, Devereux G. Diet and asthma: nutrition implications from prevention to treatment. J Am Diet Assoc 2011; 111: 258-268.

31 Beckhaus AA, Garcia-Marcos L, Forno E, et al. Maternal nutrition during pregnancy and risk of asthma, wheeze, and atopic diseases during childhood: a systematic review and meta-analysis. Allergy 2015; 70: 1588-1604

32 Smith GD. Assessing intrauterine influences on offspring health outcomes: can epidemiological studies yield robust findings? Basic Clin Pharmacol Toxicol 2008; 102: 245-256.

33 Shaheen SO, Newson RB, Smith GD, et al. Prenatal paracetamol exposure and asthma: further evidence against confounding. Int J Epidemiol 2010; 39: 790-794.

34 Maslova E, Rytter D, Bech BH, et al. Maternal protein intake during pregnancy and offspring overweight $20 \mathrm{y}$ later. Am J Clin Nutr 2014; 100: 1139-1148.

35 Hernán MA, Hernandez-Diaz S, Robins JM. A structural approach to selection bias. Epidemiology 2004; 15: 615-625.

36 Piantadosi S, Byar DP, Green SB. The ecological fallacy. Am J Epidemiol 1988; 127: 893-904.

37 Aeberli I, Gerber PA, Hochuli M, et al. Low to moderate sugar-sweetened beverage consumption impairs glucose and lipid metabolism and promotes inflammation in healthy young men: a randomized controlled trial. Am Soc Nutr 2011; 94: 479-485

38 Johnson RJ, Nakagawa T, Sanchez-Lozada LG, et al. Sugar, uric acid, and the etiology of diabetes and obesity. Diabetes 2013; 62: 3307-3315.

39 Kool M, Willart MAM, van Nimwegen M, et al. An unexpected role for uric acid as an inducer of T helper 2 cell immunity to inhaled antigens and inflammatory mediator of allergic asthma. Immunity 2011; 34: 527-540.

40 Payne AN, Chassard C, Lacroix C. Gut microbial adaptation to dietary consumption of fructose, artificial sweeteners and sugar alcohols: implications for host-microbe interactions contributing to obesity. Obes Rev 2012; 13: 799-809.

41 McLoughlin RM, Mills KHG. Influence of gastrointestinal commensal bacteria on the immune responses that mediate allergy and asthma. J Allergy Clin Immunol 2011; 127: 1097-1107.

42 Thorburn AN, McKenzie CI, Shen S, et al. Evidence that asthma is a developmental origin disease influenced by maternal diet and bacterial metabolites. Nat Commun 2015; 6: 7320.

43 Kumar R, Ouyang F, Story RE, et al. Gestational diabetes, atopic dermatitis, and allergen sensitization in early childhood. J Allergy Clin Immunol 2009; 124: 1031-1038.

44 Silverman BL, Rizzo T, Green OC, et al. Long-term prospective evaluation of offspring of diabetic mothers. Diabetes 1991; 40: Suppl. 2, 121-125.

45 Lakhan SE, Kirchgessner A. The emerging role of dietary fructose in obesity and cognitive decline. Nutr J 2013; 12: 114.

46 Robins JM, Greenland S. Identifiability and exchangeability for direct and indirect effects. Epidemiology 1992; 3 : 143-155.

47 Cole SR, Hernán MA. Fallibility in estimating direct effects. Int J Epidemiol 2002; 31: $163-165$.

48 Yarnell JW, Fehily AM, Milbank JE, et al. A short dietary questionnaire for use in an epidemiological survey: comparison with weighed dietary records. Hum Nutr Appl Nutr 1983; 37: 103-112.

49 Howe LD, Tilling K, Galobardes B, et al. Loss to follow-up in cohort studies: bias in estimates of socioeconomic inequalities. Epidemiology 2013; 24: 1-9. 